\title{
Gender-related differences in men and women with ST-segment elevation myocardial infarction and incomplete infarct-related artery flow restoration: a multicenter national registry
}

\author{
Małgorzata Zachura ${ }^{1}$, Krzysztof Wilczek², Jacek Kurzawski ${ }^{1}$, Marek Gierlotka ${ }^{3}$, Mariusz Gąsior ${ }^{2}$, \\ Marcin Sadowski ${ }^{4,5}$ \\ ${ }^{1} 2^{\text {nd }}$ Department of Cardiology, Świętokrzyskie Cardiology Centre, Kielce, Poland \\ ${ }^{2} 3^{\text {rd }}$ Department of Cardiology, School of Medicine with the Division of Dentistry in Zabrze, Medical University of Silesia in Katowice, \\ Silesian Centre for Heart Disease, Zabrze, Poland \\ ${ }^{3}$ Department of Cardiology, Faculty of Natural Sciences and Technology, University of Opole, University Hospital, Opole, Poland \\ ${ }^{4}$ Faculty of Medicine and Health Sciences, Jan Kochanowski University, Kielce, Poland \\ ${ }^{5}$ Department of Interventional Cardiology, Świętokrzyskie Cardiology Centre, Kielce, Poland
}

Adv Interv Cardiol 2018; 14, 4 (54): 356-362

DOI: https://doi.org/10.5114/aic.2018.79865

\begin{abstract}
A bstract
Introduction: Little is known about gender-related differences in ST-segment elevation myocardial infarction (STEMI) and incomplete infarct-related artery (IRA) reperfusion after primary percutaneous coronary intervention ( $\mathrm{pPCl})$.

Aim: To evaluate gender-related differences in clinical characteristics and prognosis in patients with STEMI and incomplete IRA reperfusion after $\mathrm{pPCl}$.

Material and methods: From 42,752 STEMI patients hospitalized between 2009 and 2011 in Poland we analyzed a group of 984 (36\%) females and 1,746 (64\%) males with less than Thrombolysis in Myocardial Infarction (TIMI) grade 3 flow following pPCI.

Results: Women were older than men $(72.0 \pm 11.3$ vs. $64.0 \pm 11.7$ years; $p<0.0001)$ and in age-adjusted analysis they were more likely to present with hypertension ( $73.7 \%$ vs. $67 \% ; p=0.0003)$, diabetes ( $33 \%$ vs. $22.6 \% ; p<0.0001)$ and obesity $(28.1 \%$ vs. $22.6 \% ; p=0.0016$ ). Heart rate $>100$ beats/min was more common in women, while men were more often smokers and presented with sudden cardiac arrest. The most common IRA in women was the left anterior descending artery, and the right coronary artery in men. After adjusting for age statistically significant differences in pharmacotherapy concerned only the use of insulin (OR $=1.31$, $95 \% \mathrm{Cl}: 1.02-1.68)$. High risk of death, rehospitalization due to heart failure or cardiac causes, were observed in all patients during the 6-month and 12-month follow-up periods. The risk of heart failure was significantly higher in women than in men. The most significant decrease in survival rates was observed in the in-hospital period.

Conclusions: Among patients with STEMI and post-interventional TIMI flow grade $<3$ women have unfavorable baseline characteristics and an adverse short- and long-term prognosis when compared to men.
\end{abstract}

Key words: primary percutaneous coronary intervention, thrombolysis in myocardial infarction, ST-segment myocardial infarction, gender-related differences.

Su m m a ry

Patients with ST-segment elevation myocardial infarction and incomplete infarct-related artery reperfusion after primary percutaneous coronary intervention remain a poorly studied subset. Presented research clearly highlights the gender discrepancy in baseline characteristics, short- and long-term prognosis among patients with ST-segment elevation myocardial infarction and postprocedural suboptimal blood flow. This suggests the need for gender-tailored techniques to minimize postintervention complications.

\section{Corresponding author:}

Małgorzata Zachura, 2 ${ }^{\text {nd }}$ Department of Cardiology, Świętokrzyskie Cardiology Centre, 45 Grunwaldzka St, 25-736 Kielce, Poland, phone: +48 4136715 08, e-mail: malgorzatazachura@gmail.com

Received: 24.05.2018, accepted: 13.08.2018. 


\section{Introduction}

Primary percutaneous coronary intervention $(\mathrm{pPCl})$ is the treatment of choice in patients with acute ST-segment elevation myocardial infarction (STEMI) [1]. The main goal of the primary angioplasty is the rapid and sustained blood flow restoration in the infarct-related artery (IRA). In the mid-1980s, the Thrombolysis In Myocardial Infarction (TIMI) Study Group proposed a scoring system (from 0 to 3 ) referring to levels of epicardial coronary blood flow assessed during PCI [2]. The no-reflow phenomenon referring to $\mathrm{TIMI}$ grade $<3$ is not so rare as previously thought. It is estimated that $5 \%$ to $23 \%$ of patients with STEMI fail to achieve TIMI grade 3 flow in the IRA after $\mathrm{pPCI}$ [3]. While incomplete reperfusion is an undisputed unfavorable predictor of mortality, there are conflicting reports about the impact of gender on the outcomes in STEMI patients. Some researchers consider women with STEMI as a special patient subset that requires specific attention due to their worse clinical presentation and prognosis compared to men [4]. Other studies suggest that unfavorable differences in outcomes among females mainly originate from their older age, rather than from gender itself $[5,6]$.

\section{Aim}

The purpose of the present study is therefore to analyze differences in clinical characteristics and prognosis between men and women with STEMI undergoing $\mathrm{PPCl}$ with final TIMI grade < 3. Currently, there are no convincing data on this specific patient subgroup, although they remain an important and challenging subset in clinical practice.

\section{Material and methods}

Study population Data derive from the prospective, nationwide Polish Registry of Acute Coronary Syndromes (PL-ACS) implemented by the Silesian Centre for Heart Diseases and the Polish Ministry of Health. Its methodology and an analysis of the first 100,193 patients have been previously described [7]. In practice, the registry is an ongoing, multicenter, observational study of consecutively hospitalized Polish patients representing the entire ACS spectrum (unstable angina, NSTEMI, or STEMI). A detailed protocol with inclusion and exclusion criteria, methods and logistics, and definitions of all the fields in the registry dataset had been precisely defined before the registry was launched. Patients are continuously enrolled after the diagnosis of acute coronary syndrome (ACS) is confirmed. In the current study we analyzed a total of 42,752 STEMI patients hospitalized between 2009 and 2011 in Poland. The selected group consisted of 2,730 patients with less than TIMI grade 3 flow following $\mathrm{pPCl}$. Patients with cardiogenic shock on admission were excluded. There were 948 (36\%) women and 1,746
(64\%) men in the analyzed group. Baseline characteristics, pharmacotherapy, complications during hospitalization, in-hospital and long-term mortality, were compared between women and men. The primary end-points were in-hospital, 30-day, 6-month and 12-month mortality. Major adverse cardiac events (MACE) were defined as cardiovascular death, myocardial infarction, stroke, or major bleeding. The follow-up data were obtained from the National Health Fund (NHF) and updated for all subjects included.

Data definitions According to the protocol, STEMI was defined as the presence of serum cardiac necrosis markers together with the ST-segment elevation consistent with myocardial infarction (MI) of $\geq 2 \mathrm{~mm}$ in adjacent chest leads and/or ST-segment elevation of $\geq 1 \mathrm{~mm}$ in two or more standard leads or new left bundle branch block (LBBB).

\section{Statistical analysis}

Continuous variables are presented as mean \pm standard deviation (SD) and compared by Student's $t$-test. The quantitative data were presented as numbers and percentages and compared with the $\chi^{2}$ test. Additionally, age-adjusted odds ratios with confidence intervals for women vs. men were calculated for each parameter using logistic regression. Follow-up mortality was analyzed using the Kaplan-Meier method and the log-rank test. Variables with a $p$-value $<0.05$ were considered statistically significant. Calculations were performed using NCSS 12 Statistical Software (NCSS, LLC. Kaysville, Utah, USA) and R statistical package, version 2.15.3 (R Development Team, Vienna, Austria).

\section{Results}

Out of 40,637 patients with STEMI 34,457 (92.3\%) were treated with $\mathrm{pPCl}$. In this group, 2,730 (7.9\%) patients achieved less than TIMI grade 3 post-procedural flow. The numbers of patients with particular TIMI grades were as follows: 753 (2.2\%) TIMI 0, 414 (1.2\%) TIMI 1 and 1563 (4.5\%) TIMI 2. There was no gender-specific difference in the incidence of individual TIMI grades.

\section{Clinical and angiographic characteristics of patients}

It was found that women were older than men by an average of 8 years. Moreover, they were more likely to present well-known risk factors such as hypertension, diabetes, and obesity. Men were more often smokers and had a history of $\mathrm{MI}$ and coronary artery bypass grafting (CABG). There were no gender-related differences in the time to presentation to the emergency room after symptom onset. However, most of the patients presented to the emergency room 3-12 h since symptoms onset. In men, cardiac arrest was significantly more common at admission, while tachycardia occurred more often in females. 
After adjustment for age, female gender was no longer an independent risk factor for hyperlipidaemia, atrial fibrillation (AF), heart rate > 100 beats/min, anterior $\mathrm{MI}$, and sudden cardiac arrest (SCA) during hospitalization.

The vessel responsible for myocardial infarction was most commonly the left anterior descending (LAD) artery in women, whereas it was the right coronary artery (RCA) or an aorto-coronary bypass in men. However, the latter turned out to be irrelevant after the age-adjusted analysis (odds ratio (OR) for aorto-coronary bypass occlusion in men: $0.5,95 \%$ confidence interval $(\mathrm{Cl})$ : 0.23-1.12).
Culprit lesions were identified in 1726 men and in 931 women. Other cases were considered as MI with no obstructive coronary artery disease (MINOCA, Table I).

\section{In-hospital complications}

There was no gender-related difference in the incidence of: myocardial infarction $(0.7 \%$ in males vs. $0.8 \%$ in females, $p=0.71$ ), pulmonary edema ( $3 \%$ vs. $3.4 \%$, $p=0.59)$, stroke (0.5 vs. $0.7 \%$ in females, $p=0.39)$, intracranial hemorrhage ( $0 \%$ vs. $0.1 \%, p=0.77$ ) or target lesion revascularization during hospitalization (1.4\% vs.

Table I. Baseline characteristics

\begin{tabular}{|c|c|c|c|c|}
\hline Parameter & $\begin{array}{c}\text { Men } \\
1746(64 \%)\end{array}$ & $\begin{array}{c}\text { Women } \\
984(36 \%)\end{array}$ & $P$-value & $\begin{array}{l}\text { Age-adjusted odds ratio } \\
\text { for females }(95 \% \mathrm{Cl})\end{array}$ \\
\hline Age, mean \pm SD & $64.0 \pm 11.7$ & $72.0 \pm 11.3$ & $<0.0001$ & - \\
\hline Older than 65 years & $807(46.2 \%)$ & $734(74.6 \%)$ & $<0.0001$ & - \\
\hline Age $<65$ years & $939(53.8 \%)$ & $250(25.4 \%)$ & $<0.0001$ & - \\
\hline Arterial hypertension & $1170(67 \%)$ & $725(73.7 \%)$ & 0.0003 & $1.22(1.02-1.47)$ \\
\hline Diabetes & $394(22.6 \%)$ & $325(33 \%)$ & $<0.0001$ & $1.55(1.29-1.86)$ \\
\hline Hyperlipidemia & $690(39.5 \%)$ & $389(39.5 \%)$ & 0.99 & $1.09(0.92-1.29)$ \\
\hline Ever smoked & $1238(70.9 \%)$ & $337(34.3 \%)$ & $<0.0001$ & $0.28(0.24-0.34)$ \\
\hline Obesity (BMI > $\left.30 \mathrm{~kg} / \mathrm{m}^{2}\right)$ & $395(22.6 \%)$ & $276(28.1 \%)$ & 0.0016 & $1.54(1.27-1.86)$ \\
\hline Renal failure & $106(6.1 \%)$ & $74(7.5 \%)$ & 0.14 & $0.84(0.61-1.17)$ \\
\hline Previous myocardial infarction & $269(15.4 \%)$ & $115(11.7 \%)$ & 0.0073 & $0.68(0.53-0.87)$ \\
\hline Previous $\mathrm{PCl}$ & $174(10 \%)$ & $66(6.7 \%)$ & 0.0039 & $0.76(0.56-1.03)$ \\
\hline Previous CABG & $50(2.9 \%)$ & $10(1 \%)$ & 0.0016 & $0.4(0.2-0.8)$ \\
\hline Congestive heart failure & $104(6 \%)$ & $79(8 \%)$ & 0.038 & $0.99(0.73-1.37)$ \\
\hline \multicolumn{5}{|l|}{ Dominant symptom type: } \\
\hline Chest pain & $1601(91.7 \%)$ & $903(91.8 \%)$ & 0.95 & $1.07(0.79-1.44)$ \\
\hline Dyspnea & $34(2 \%)$ & $27(2.7 \%)$ & 0.18 & $1.28(0.74-2.2)$ \\
\hline Cardiac arrest & $43(2.5 \%)$ & $10(1 \%)$ & 0.0085 & $0.46(0.23-0.95)$ \\
\hline \multicolumn{5}{|l|}{ EKG on admission: } \\
\hline Sinus rhythm & $1587(90.9 \%)$ & $841(85.5 \%)$ & $<0.0001$ & $0.86(0.67-1.11)$ \\
\hline $\mathrm{AF}$ & $124(7.1 \%)$ & $115(11.7 \%)$ & $<0.0001$ & $1.14(0.86-1.52)$ \\
\hline $\mathrm{HR}>100 \mathrm{~min}^{-1}$ & $179(10.3 \%)$ & $143(14.5 \%)$ & 0.0009 & $1.45(1.13-1.86)$ \\
\hline \multicolumn{5}{|l|}{ Infarct location: } \\
\hline Anterior & 713 (40.8\%) & $457(46.4 \%)$ & 0.0045 & $1.15(0.98-1.36)$ \\
\hline Inferior & $865(49.5 \%)$ & $426(43.3 \%)$ & 0.0017 & $0.83(0.71-0.98)$ \\
\hline \multicolumn{5}{|c|}{ Symptom onset-to-admission time: } \\
\hline $0-3 \mathrm{~h}$ & 567 (33.8\%) & 297 (31.5\%) & 0.23 & $0.93(0.78-1.11)$ \\
\hline $3-12 \mathrm{~h}$ & $679(40.5 \%)$ & $390(41.4 \%)$ & 0.66 & $1.00(0.85-1.19)$ \\
\hline$>12 \mathrm{~h}$ & $432(25.7 \%)$ & $256(27.2 \%)$ & 0.43 & $1.08(0.89-1.3)$ \\
\hline \multicolumn{5}{|l|}{ Infarct-related artery: } \\
\hline $\mathrm{LM}$ & $29(1.7 \%)$ & $13(1.3 \%)$ & 0.49 & $0.8(0.4-1.59)$ \\
\hline LAD & $679(38.9 \%)$ & $463(47.1 \%)$ & $<0.0001$ & $1.29(1.09-1.52)$ \\
\hline RCA & $727(41.6 \%)$ & $353(35.9 \%)$ & 0.0031 & $0.83(0.7-0.99)$ \\
\hline SVG & $31(1.8 \%)$ & $8(0.8 \%)$ & 0.042 & $0.5(0.23-0.12)$ \\
\hline \multicolumn{5}{|l|}{ Post-procedural TIMI grade: } \\
\hline TIMI 2 & $1021(58.5 \%)$ & $542(55.1 \%)$ & 0.085 & $0.93(0.78-1.09)$ \\
\hline TIMI 0 or 1 & 725 (41.5\%) & $442(44.9 \%)$ & 0.085 & $1.08(0.91-1.28)$ \\
\hline
\end{tabular}

$B M I$ - body mass index, $C l$ - confidence interval, $P C l$ - percutaneous coronary intervention, CABG - coronary artery bypass surgery, EKG - electrocardiogram, $A F-$ atrial fibrillation, HR - heart rate, LM - left main coronary artery, LAD - left anterior descending artery, RCA - right coronary artery, TIMI - Thrombolysis in Myocardial Infarction, SVG - saphenous vein graft. 
$1.2 \%, p=0.73)$. Sudden cardiac death, bleeding, acute mitral regurgitation (AMR), free wall rupture (FWR) and infarct-related ventricular septal defect (VSD) were more common in females. After adjusting for age, the only significant complication occurring more often in women was VSD (Table II).

\section{On-admission pharmacotherapy}

In the primary analysis, women were less likely to receive acetylsalicylic acid (ASA), glycoprotein (GP) Ilb/ Illa inhibitors, angiotensin-converting enzyme inhibitors (ACEI), $\beta$-blockers and statins, while the usage of diuretics and insulin was more common in women than in men. After adjusting for age, statistically significant differences concerned only the use of insulin $(O R=1.31$, $95 \% \mathrm{Cl}: 1.02-1.68)$. In $9.4 \%$ of men and $11.9 \%$ of women ASA was not used (Table III).

\section{Follow-up}

High risk of-death, rehospitalization due to heart failure or cardiac causes and MACE were observed in all patients during the 6-month and 12-month follow-up periods. After age adjustment the risk of heart failure was significantly higher in women than in men (Table IV).

\section{In-hospital and long-term mortality}

The mortality was significantly higher in women than in men in each follow-up period. The most significant de- crease in survival rates was observed in the in-hospital period (Figure 1).

\section{Discussion}

The major finding of our study is that women with STEMI and post-procedural suboptimal coronary blood flow have unfavorable clinical presentation and higher mortality rate in each follow-up period, compared to men. To the best of our knowledge, this is the first report on gender-related differences in a large population of STEMI patients with incomplete reperfusion, as many other published papers focused on determining the predictive factors of suboptimal flow after $\mathrm{pPCl}$ with no gender specification [3, 8]. Moreover, available data comparing outcomes in men and women with STEMI remain controversial, with some authors reporting worse prognosis in females and others showing no sex differences in clinical endpoints $[4,9,10]$. These discrepancies can be partly explained by different inclusion criteria and size of study populations [10]. However, it seems that the most confounding factor when comparing the outcomes of men versus women with STEMI is age. Existing data indicates a potential interaction between age and gender as in most studies females were older than men and thus had more comorbidities affecting overall results [11]. Similarly, in our study women with suboptimal blood flow after $\mathrm{pPCl}$ were older than men by an average of 8 years and had more well-known risk factors for cardiovascu-

Table II. In-hospital complications

\begin{tabular}{lcccc} 
Parameter & $\begin{array}{c}\text { Men } \\
\mathbf{1 7 4 6 ( 6 4 \% )}\end{array}$ & $\begin{array}{c}\text { Women } \\
\mathbf{9 8 4}(\mathbf{3 6 \% )}\end{array}$ & $\begin{array}{c}\boldsymbol{P} \text {-value } \\
\text { Age-adjusted odds ratio } \\
\text { for females (95\% CI) }\end{array}$ \\
\hline Bleeding & $40(2.3 \%)$ & $40(4.1 \%)$ & 0.0083 & $7.31(0.82-2.1)$ \\
\hline AMR & $1(0.1 \%)$ & $5(0.5 \%)$ & 0.047 & 0.027 \\
\hline FWR & $5(0.3 \%)$ & $9(0.9 \%)$ & $0.88-68.73)$ & $2.43(0.78-7.55)$ \\
\hline VSD & $2(0.1 \%)$ & $8(0.8 \%)$ & 0.0003 & $7.4(1.49-36.87)$ \\
\hline Cardiac death & $201(11.5 \%)$ & $162(16.5 \%)$ & $1.05(0.83-1.34)$
\end{tabular}

$\mathrm{Cl}$-confidence interval, AMR - acute mitral regurgitation, FWR - free wall rupture, VSD - ventricular septal defect.

Table III. In-hospital pharmacotherapy

\begin{tabular}{|c|c|c|c|c|}
\hline Parameter & $\begin{array}{c}\text { Men } \\
1746(64 \%) \\
\end{array}$ & $\begin{array}{l}\text { Women } \\
984(36 \%)\end{array}$ & $P$-value & $\begin{array}{l}\text { Age-adjusted odds ratio } \\
\text { for females }(95 \% \mathrm{Cl})\end{array}$ \\
\hline Acetylsalicylic acid & $1582(90.6 \%)$ & $867(88.1 \%)$ & 0.039 & $0.83(0.64-1.08)$ \\
\hline Clopidogrel & $1725(98.8 \%)$ & $970(98.6 \%)$ & 0.62 & $0.9(0.44-1.85)$ \\
\hline GP IIb/IIla inhibitor & $725(41.5 \%)$ & $350(35.6 \%)$ & 0.0022 & $0.92(0.78-1.1)$ \\
\hline$\beta$-Blocker & $1274(73 \%)$ & $660(67.1 \%)$ & 0.0011 & $0.86(0.72-1.03)$ \\
\hline ACEI & $1170(67 \%)$ & $610(62 \%)$ & 0.0082 & $0.9(0.76-1.07)$ \\
\hline ARB & $34(2 \%)$ & $15(1.5 \%)$ & 0.42 & $0.73(0.38-1.37)$ \\
\hline Statin & $1398(80.1 \%)$ & $752(76.4 \%)$ & 0.025 & $0.9(0.74-1.11)$ \\
\hline Diuretics & $374(21.4 \%)$ & $278(28.3 \%)$ & $<0.0001$ & $1.13(0.93-1.37)$ \\
\hline Aldosterone antagonist & $146(8.4 \%)$ & $102(10.4 \%)$ & 0.080 & $1.22(0.92-1.61)$ \\
\hline Insulin & $182(10.4 \%)$ & 139 (14.1\%) & 0.0039 & $1.31(1.02-1.68)$ \\
\hline Oral antihyperglycemic agents & $92(5.3 \%)$ & $57(5.8 \%)$ & 0.56 & $1.04(0.73-1.48)$ \\
\hline
\end{tabular}

$\mathrm{Cl}$ - confidence interval, GP-glycoprotein, ACEI - angiotensin-converting enzyme inhibitor, ARB - angiotensin receptor blocker. 
Table IV. Follow-up of patients with STEMI and post-procedural TIMI grade $<3$

\begin{tabular}{|c|c|c|c|c|}
\hline Parameter & $\begin{array}{c}\text { Men } \\
1453(65 \%) \\
\end{array}$ & $\begin{array}{l}\text { Women } \\
766(35 \%)\end{array}$ & $P$-value & $\begin{array}{l}\text { Age-adjusted odds ratio } \\
\text { for females }(95 \% \mathrm{Cl})\end{array}$ \\
\hline \multicolumn{5}{|l|}{ Six-month follow-up: } \\
\hline Death & $135(9.3 \%)$ & $113(14.8 \%)$ & 0.0001 & $1.08(0.89-1.32)$ \\
\hline Reinfarction & $46(3.2 \%)$ & $27(3.5 \%)$ & 0.65 & $1.09(0.66-1.8)$ \\
\hline Heart failure rehospitalization & $96(6.6 \%)$ & $97(12.7 \%)$ & $<0.0001$ & $1.5(1.1-2.03)$ \\
\hline Cardiac causes rehospitalization & $603(41.5 \%)$ & $300(39.2 \%)$ & 0.29 & $0.94(0.78-1.12)$ \\
\hline MACE & $698(48 \%)$ & $380(49.6 \%)$ & 0.48 & $1.01(0.85-1.2)$ \\
\hline \multicolumn{5}{|l|}{ Twelve-month follow-up: } \\
\hline Death & $163(11.2 \%)$ & $144(18.8 \%)$ & $<0.0001$ & $1.16(0.96-1.4)$ \\
\hline Reinfarction & $61(4.2 \%)$ & $37(4.8 \%)$ & 0.49 & $1.08(0.7-1.67)$ \\
\hline Heart failure rehospitalization & $129(8.9 \%)$ & $113(14.8 \%)$ & $<0.0001$ & $1.33(1.0-1.75)$ \\
\hline Cardiac cause rehospitalization & $689(47.4 \%)$ & $359(46.9 \%)$ & 0.80 & $1.01(0.85-1.2)$ \\
\hline MACE & 791 (54.4\%) & 448 (58.5\%) & 0.068 & $1.11(0.93-1.32)$ \\
\hline
\end{tabular}

$\mathrm{Cl}$-confidence interval, MACE - major adverse cardiac events.

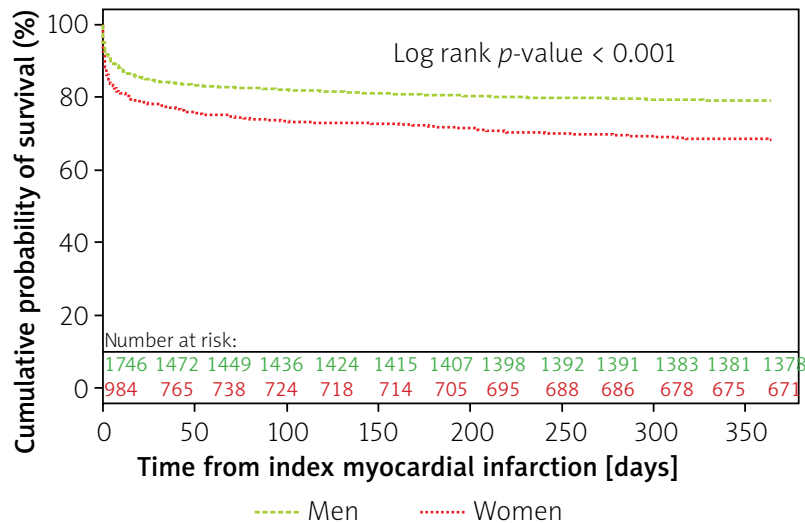

Figure 1. Kaplan-Meier survival curves in patients with STEMI and post-procedural TIMI $<3$

lar disease. In addition, some of these factors such as hypertension, diabetes and obesity still occurred more often in women after an age-adjusted analysis. Comorbidities and advanced age deeply influence the clinical presentation. This is confirmed by the fact that among all the analyzed factors after the age-adjusted analysis, only tachycardia occurred more frequently in women. Although it is well documented that women with STEMI have time delays in medical help seeking longer than their male counterparts [12], we did not observe gender-related differences in the symptom onset-to-balloon time in the studied population.

In our study, the incidence of suboptimal coronary blood flow was $7.9 \%$, and it is comparable with other studies which similarly used the criterion of TIMI flow grade $<3[3,13,14]$. In a multivariate analysis Gąsior et al. found that diabetes and advanced age were independent determinants of post-procedural coronary flow in patients with myocardial infarction [15]. Data analyses from the Primary Angioplasty in Myocardial Infarction (PAMI) trials also revealed age $>70$ years, diabetes and in addition heart failure as important risk factors for final TIMI flow grade $<3$ after $\mathrm{pPCI}$ [3]. None of the mentioned studies have shown that female sex was a predictor of interventional treatment failure. However, women have narrower coronary arteries than males [16] and it might be considered as a possible obstacle in balloon or stent delivery. According to Watanabe et al., the ratio of stent diameter to vessel diameter (per 0.1 increase) is significantly associated with slow flow following stent implantation [17]. Moreover, low estrogen levels in post-menopausal women contribute to the development of coronary microvascular disease [18] and the no-reflow phenomenon. Some researchers indicate that patients with the LAD as the IRA have fourfold greater risk of the no-reflow phenomenon $[15,19]$. As it was previously investigated, acute $M I$ involving the LAD is associated with worse prognosis due to lower ejection fraction and risk of developing heart failure [20]. Consequently, anterior STEMI increases the risk of death and post-infarction heart failure [21]. In our study, LAD occlusion was more common in women, which could contribute to other findings regarding their worse prognosis compared to men.

Our study showed that women with STEMI and incomplete revascularization have more in-hospital complications. Although previous studies indicate that females after $\mathrm{PPCl}$ have more bleeding complications compared to men $[22,23]$, we did not observe such dependence after adjustment for age. Surprisingly, some complications in the studied group occurred less frequently than in the whole population of patients with STEMI, applying to FWR or VSD [24].

Based on the results, women are less likely to receive guideline-recommended pharmacotherapy during hospitalization. However, these differences are mainly age-dependent as women are almost a decade older than men, with higher prevalence of hemorrhagic complications, diabetes mellitus and renal failure. There are no spe- 
cific data to clarify the reason for the surprisingly low percentage of ASA usage; however, we hypothesize that some patients have already used chronic anticoagulation for other reasons such as an artificial heart valve, atrial fibrillation or a history of pulmonary embolism. Moreover, certain patients could have contraindications for ASA administration because of allergy. Similarly, Akhter et al. showed that women after ACS were less likely to receive aspirin and GP IIb/IIla inhibitors, and were less often discharged on aspirin [23]. Several large studies evaluating gender-related differences in STEMI patients also revealed that men receive optimal therapy more often than women (including interventional treatment) $[25,26]$. According to our data, $7.7 \%$ of females did not undergo $\mathrm{pPCl}$. Although the registry does not allow us to precisely identify the causes, we suspect that it could be explained by MINOCA, subsequent CABG or coronary lesions not amenable to revascularization. No specific analyses were performed among patients with MINOCA as due to the small sample size (20 men and 13 women) they were included in the entire studied population. However, new guidelines of the European Society of Cardiology (ESC) emphasize that MINOCA comprises up to $14 \%$ of STEMI patients and requires additional diagnostic tests and tailored therapy which may differ from typical STEMI management [27].

Previous studies clearly indicate that TIMI grade $<3$ flow after $\mathrm{pPCl}$ in patients with $\mathrm{MI}$ leads to poor in-hospital and long-term outcomes [3, 13, 28]. Based on PAMI trials, patients who did not achieve optimal TIMI flow after $\mathrm{pPCl}$ had significantly higher rates of in-hospital mortality and in-hospital major adverse cardiac events than those with TIMI grade 3 [3]. Likewise, an increase in early (30-day) mortality with TIMI grade $<3$ flow was noted in the GUSTO-IIb trial [29]. Another study by Champney et al. revealed that 30-day mortality after STEMI was higher among women and even after adjusting for comorbidities mortality remained about $15-20 \%$ higher in younger females than in men [11]. In the present study, after adjustment for age we did not observe a statistically significant difference in mortality between the sexes. Moreover, the survival rates decreased substantially in the early period after STEMI, and then remained moderately stable. Additionally, during the 12-month follow-up period we observed higher risk of heart failure in women, which may be associated with more frequent microvascular and endothelial dysfunction [30].

\section{Study limitations}

Although the PL-ACS Registry is a prospective observational study, not all hospitals treating ACS patients in Poland contributed to data collection. Data also vary depending on the center and country region. Thus, the data averaged for Poland may not necessarily reflect the actu- al situation in the different regions of the country. Moreover, the imaging protocol was not standardized in individual catheterization laboratories. The catheterization centers participating in the study did not have a core laboratory and therefore the TIMI grade assessment was operator-dependent. However, experimental studies prove the close correlation of individual TIMI grades with the myocardial necrosis area even if the assessment is often subjective. In the present study the effect of vascular access was not analyzed.

\section{Conclusions}

Our study shows that among patients with STEMI and incomplete epicardial coronary blood flow restoration following $\mathrm{pPCl}$ women have unfavorable baseline characteristics and clinical presentation. They also have an adverse short- and long-term prognosis when compared to men, including higher risk of heart failure development. After adjustment for age the in-hospital and long-term mortality is similar in both sexes, with the greatest decrease in survival during the in-hospital period.

\section{Conflict of interest}

The authors declare no conflict of interest.

\section{References}

1. The Task Force on the management of ST-segment elevation acute myocardial infarction of the European Society of Cardiology (ESC). ESC Guidelines for the management of acute myocardial infarction in patients presenting with ST-segment elevation. Eur Heart J 2012; 33: 2569-619.

2. TIMI Study Group. The Thrombolysis In Myocardial Infarction (TIMI) trial. N Engl J Med 1985; 31: 932-6.

3. Mehta RH, Harjai KJ, Cox D, et al.; Primary Angioplasty in Myocardial Infarction (PAMI) Investigators. Clinical and angiographic correlates and outcomes of suboptimal coronary flow in patients with acute myocardial infarction undergoing primary percutaneous coronary intervention. J Am Coll Cardiol 2003; 42: 1739-46.

4. Kytö V, Sipilä J, Rautava P. Gender and in-hospital mortality of ST-segment elevation myocardial infarction (from a multihospital nationwide registry study of 31,689 patients). Am J Cardiol 2015; 115: 303-6.

5. Park JS, Kim YJ, Shin DG, et al. Gender differences in clinical features and inhospital outcomes in ST-segment elevation acute myocardial infarction: from the Korean acute myocardial infarction registry (KAMIR) study. Clin Cardiol 2010; 33: E1-6.

6. Ghaffari S, Pourafkari L, Tajlil A, et al. Is female gender associated with worse outcome after ST elevation myocardial infarction? Indian Heart J 2017; 69 Suppl 1: S28-33.

7. Poloński L, Gąsior M, Gierlotka M, et al. Polish Registry of Acute Coronary Syndromes (PL-ACS). Characteristics treatments and outcomes of patients with acute coronary syndromes in Poland. Kardiol Pol 2007; 65: 861-72.

8. Shiraishi J, Kohno Y, Sawada T, et al. Predictors of nonoptimal coronary flow after primary percutaneous coronary intervention with stent implantation for acute myocardial infarction. J Cardiol 2010; 55: 217-23. 
9. Barthélémy O, Degrell P, Berman E. Sex-related differences after contemporary primary percutaneous coronary intervention for ST-segment elevation myocardial infarction. Arch Cardiovasc Dis 2015; 108: 428-36.

10. Perl L, Bental T, Assali A, et al. Impact of female sex on long-term acute coronary syndrome outcomes. Coron Artery Dis 2015; 26: $11-6$

11. Champney KP, Frederick PD, Bueno $\mathrm{H}$, et al. The joint contribution of sex, age and type of myocardial infarction on hospital mortality following acute myocardial infarction. Heart 2009; 95 : 895-9.

12. Krumholz HM, Herrin J, Miller LE, et al. Improvements in doorto-balloon time in the United States, 2005 to 2010. Circulation 2011; 124: 1038-45.

13. Kammler J, Kypta A, Hofmann R, et al. TIMI 3 flow after primary angioplasty is an important predictor for outcome in patients with acute myocardial infarction. Clin Res Cardiol 2009; 98: 165-70.

14. Piana RN, Paik GY, Moscucci M, et al. Incidence and treatment of 'no-reflow' after percutaneous coronary intervention. Circulation 1994; 89: 2514-8.

15. Gąsior M, Pres D, Gierlotka M. Factors affecting microvascular flow in patients with myocardial infarction treated with percutaneous coronary interwention. Post Kardiol Interw 2007; 3: 121-7.

16. Kornowski R, Lansky AJ, Mintz GS, et al. Comparison of men versus women in cross-sectional area luminal narrowing, quantity of plaque, presence of calcium in plaque, and lumen location in coronary arteries by intravascular ultrasound in patients with stable angina pectoris. Am J Cardiol 1997; 79: 1601-5.

17. Watanabe Y, Sakakura K, Taniguchi Y, et al. Determinants of slow flow following stent implantation in intravascular ultrasound-guided primary percutaneous coronary intervention. Heart Vessels 2018; 33: 226-38.

18. Yi Xu, Ivan A, Arenas Stephen A, et al. Estrogen improves cardiac recovery after ischemia/reperfusion by decreasing tumor necrosis factor alpha. Cardiovasc Res 2006; 69: 836-44.

19. Prasad A, Stone GW, Stuckey TD, et al. Impact of diabetes mellitus on myocardial perfusion after primary angioplasty in patients with acute myocardial infarction. J Am Coll Cardiol 2005; 45: 508-14.

20. Kandzari DE, Tcheng JE, Gersh BJ, et al. Relationship between infarct artery location, epicardial flow, and myocardial perfusion after primary percutaneous revascularization in acute myocardial infarction. Am Heart J 2006; 151: 1288-95.

21. Petrina M, Goodman SG, Eagle KA. The 12-lead electrocardiogram as a predictive tool of mortality after acute myocardial infarction: current status in an era of revascularization and reperfusion. Am Heart J 2006; 152: 11-8.

22. Numasawa Y, Kohsaka S, Ueda I, et al. Incidence and predictors of bleeding complications after percutaneous coronary intervention. J Cardiol 2017; 69: 272-9.

23. Akhter N, Milford-Beland S, Roe MT, et al. Gender differences among patients with acute coronary syndromes undergoing percutaneous coronary intervention in the American College of Cardiology-National Cardiovascular Data Registry (ACC-NCDR). Am Heart J 2009; 157: 141-8.

24. Sadowski M, Janion-Sadowska $A$. The management of patients with cardiogenic shock. Med Stud 2017; 33: 55-62.

25. Kołodziej M, Kurzawski J, Janion-Sadowska A, et al. Mortality of women with ST-segment elevation myocardial infarction and cardiogenic shock - results from the PL-ACS registry. Med Stud 2016; 32: 157-63.
26. Nguyen JT, Berger AK, Duval S, et al. Gender disparity in cardiac procedures and medication use for acute myocardial infarction. Am Heart J 2008; 155: 862-8.

27. Ibanez B, James S, Agewall S, et al. 2017 ESC Guidelines for the management of acute myocardial infarction in patients presenting with ST-segment elevation: The Task Force for the management of acute myocardial infarction in patients presenting with ST-segment elevation of the European Society of Cardiology (ESC). Eur Heart J 2018; 39: 119-77.

28. Mehta RH, Ou FS, Peterson ED, et al. Clinical significance of postprocedural TIMI flow in patients with cardiogenic shock undergoing primary percutaneous coronary intervention. J Am Coll Cardiol Cardiovasc Interv 2009; 2: 56-64.

29. Lee CH, Wong HB, Tan HC, et al. Impact of reversibility of no reflow phenomenon on 30-day mortality following percutaneous revascularization for acute myocardial infarction-insights from a 1.328 patient registry. J Interv Cardiol 2005; 18: 261.

30. Reynolds HR, Srichai MB, Iqbal SN, et al. Mechanisms of myocardial infarction in women without angiographically obstructive coronary artery disease. Circulation 2011; 124: 1414-25. 